\title{
Determining spontaneous fission properties by direct mass measurements with the FRS Ion Catcher
}

\author{
${ }^{1}$ Soreq Nuclear Research Center, Yavne 81800, Israel \\ ${ }^{2}$ Tel Aviv University, Tel Aviv 69978, Israel \\ ${ }^{3}$ GSI Helmholtzzentrum für Schwerionenforschung GmbH, 64291 Darmstadt, Germany \\ ${ }^{4}$ Justus-Liebig-Universität Gießen, 35392 Gießen, Germany \\ ${ }^{5}$ IFIN-HH/ELI-NP, 077126, Magurele - Bucharest, Romania \\ ${ }^{6}$ Université Laval, Québec G1V 0A6, Canada \\ ${ }^{7}$ KVI-CART/University of Groningen, $9700 \mathrm{AB}$ Groningen, the Netherlands \\ ${ }^{8}$ Department of Physics and Astronomy Uppsala University, SE-751 05 Uppsala, Sweden \\ ${ }^{9}$ University of Novi Sad, 21101 Novi Sad, Serbia
}

Israel Mardor ${ }^{1,2, *}$, Timo Dickel ${ }^{3,4}$, Daler Amanbayev ${ }^{4}$, Samuel Ayet San Andrés ${ }^{3,4}$, Sönke Beck ${ }^{4}$, David Benyamin ${ }^{2,}$ Julian Bergmann ${ }^{4}$, Paul Constantin ${ }^{5}$, Alexandre Cléroux Cuillerier ${ }^{4,6}$, Hans Geissel ${ }^{3,4}$, Lizzy Gröff ${ }^{4}$, Christine Hornung ${ }^{4}$, Gabriella Kripko-Koncz ${ }^{4}$, Ali Mollaebrahimi ${ }^{4,7}$, Ivan Miskun ${ }^{4}$, Wolfgang R. Plaß ${ }^{3,4}$, Stephan Pomp ${ }^{8}$, Adrian Rotaru ${ }^{5}$, Christoph Scheidenberger ${ }^{3,4}$, , Goran Stanic ${ }^{3,9}$, , Christian Will $^{4,}$, and the FRS Ion Catcher Collaboration

\begin{abstract}
We present a direct method to measure fission product yield distributions (FPY) and isomeric yield ratios (IYR) for spontaneous fission (SF) fragments. These physical properties are of utmost importance to the understanding of basic nuclear physics, the astrophysical rapid neutron capture process ('r process') of nucleosynthesis, neutron star composition, and nuclear reactor safety. With this method, fission fragments are produced by spontaneous fission from a source that is mounted in a cryogenic stopping cell (CSC), thermalized and stopped within it, and then extracted and transported to a multiple-reflection time-of-flight mass-spectrometer (MR-TOF-MS). We will implement the method at the FRS Ion Catcher (FRS-IC) at GSI (Germany), whose MRTOF-MS relative mass accuracy $\left(\sim 10^{-7}\right)$ and resolving power $(\sim 600,000$ FWHM) are sufficient to separate all isobars and numerous isomers in the fission fragment realm. The system's essential element independence and its fast simultaneous mass measurement provide a new direct way to measure isotopic FPY distributions, which is complementary to existing methods. It will enable nuclide FPY measurements in the high fission peak, which is hardly accessible by current techniques. The extraction time of the CSC, tens of milliseconds, enables a direct measurement of independent fission yields, and a first study of the temporal dependence of FPY distributions in this duration range. The ability to resolve isomers will further enable direct extraction of numerous IYRs while performing the FPY measurements. The method has been recently demonstrated at the FRS-ICr for SF with a $37 \mathrm{kBq}{ }^{252} \mathrm{Cf}$ fission source, where about 70 different fission fragments have been identified and counted. In the near future, it will be used for systematic studies of SF with a higher-activity ${ }^{252} \mathrm{Cf}$ source and a ${ }^{248} \mathrm{Cm}$ source. The method can be implemented also for neutron induced fission at appropriate facilities.
\end{abstract}

\section{Background}

Nuclear fission was discovered in 1939, and has since been extensively researched and also applied to the design and construction of nuclear reactors. Nevertheless, there is still high interest in the basic and applied nuclear science community to study the fundamentals of this process, generate new data related to fission and fission products, and develop new measurement methods.

Fission Product Yields (FPYs) and Isomer Yield Ratios (IYRs) form an integral part of the prediction of antineutrino spectra generated by nuclear reactors. Nevertheless, it has been suggested that inaccuracies in the fission data used in the evaluations of these experiments led

*e-mail: mardor@tauex.tau.ac.il to discrepancies and anomalies with respect to the best $\beta$ decay model predictions [1].

Fission processes play a significant role in the astrophysical rapid neutron capture process ('r process') of nucleosynthesis. The inclusion of spontaneous fission (SF), neutron-induced fission, beta-delayed fission and neutrino-induced fission, supplemented with realistic distributions of fission yields, leads to improved predictions of the r-process elemental abundance in the universe [2].

Though extensive data on fission exists, the International Atomic Energy Agency (IAEA) advocates new fission experimental efforts, because of existing and emerging requirements for fission data in reactor technologies, waste management and safeguards. The IAEA specifically recommends new approaches such as direct ion counting of fission fragments, especially for IYRs, which are impor- 
tant for calculations of post reactor shutdown decay heat, but their experimental data is rather limited [3]. The IAEA interest includes IYRs for SF as well [4].

Direct ion counting with a Penning trap was used at the Ion Guide Isotope Separator On-Line (IGISOL) facility at the University of Jyväskylä [5] for measuring Independent FPYs of 25 and $50 \mathrm{MeV}$ proton-induced fission of ${ }^{n a t} \mathrm{U}$ [6], and IYRs of $25 \mathrm{MeV}$ proton-induced fission of ${ }^{\text {nat }} \mathrm{U}$ and ${ }^{232} \mathrm{Th}[7,8]$.

FPYs and IYRs are also measured by other methods at the the recoil mass spectrometer Lohengrin at the Institut Laue-Langevin, Grenoble, France [9]. Unit mass separation is achieved by the recoil mass spectrometer, and isotopic fission yields are determined by ionization chambers, Z-dependent solid absorbers and gamma spectroscopy [10]. However, each of the above methods is limited to a certain nuclide region. In general, $\gamma$-ray spectroscopy is limited for very rare fission products, whose energy levels are not well-known a-priori.

Isotope FPY data is scarce due to methodical difficulties in fragment spectroscopy. For ${ }^{252} \mathrm{Cf}$ SF it exists mainly in the low fission peak $(\mathrm{A} \sim 80-125)$ due to limited resolution in $\mathrm{Z}$ [11]. Experimental data on the high fission peak is limited to 'cold fission' (i.e. without neutron emission) [12], and to an evaluation based on the measurement of two nuclides per isobar chain [13].

IYR data for ${ }^{252} \mathrm{Cf}$ SF exist for only $\sim 10$ nuclides, in a limited region $(\mathrm{A}=128-138)$ in the high fission peak [14]. It was extracted from $\gamma$-ray spectroscopy coupled with radiochemistry, relied on $\gamma$-ray branching ratios that were not always well-known, and was limited to fragments with half-lives longer than one minute.

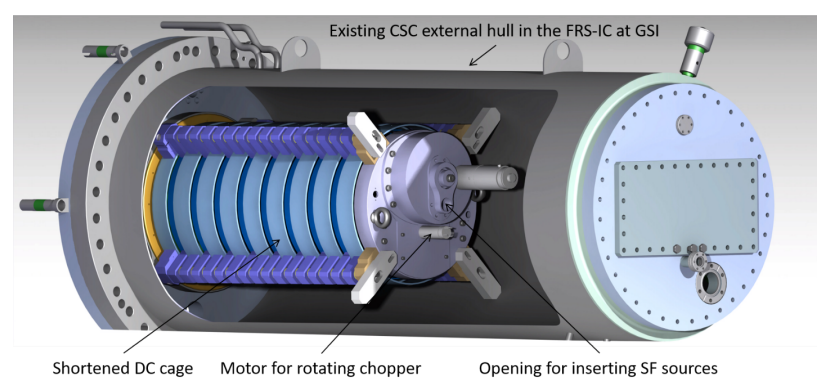

Figure 1. Drawing of the internal instrumentation that is designed for the SF experiments in the existing CSC at the FRS-IC. The shortened DC cage is seen, covering only half of the CSC hull. Further depicted is the opening for inserting SF sources, and a motor for rotating the chopper

\section{Description of the Method}

The method is realized at the FRS Ion Catcher (FRS-IC) [15], at the FRagment Separator (FRS) [16] in GSI (Germany). It usually utilizes rare isotopes from the FRS following in-flight fragmentation or fission of relativistic heavy ions. Thermalization is performed in a cryogenic stopping cell (CSC) [17-19]. The isotopes are then extracted, diagnosed and transported by a versatile RFQ beam-line [20]. Their mass is measured precisely with a multiple-reflection time-of-flight mass spectrometer (MRTOF-MS) [21] that can also provide an isobarically (and isomerically) clean beam for further experiments [22].

FPY distributions of SF are obtained by installing an SF source in the CSC, identifying the fission fragments (including isomers) in the MR-TOF-MS mass spectra, and counting the amount of each identified nuclide. Usage of SF fragments by installing a SF source in a gas cell has been realized before at "Miss Piggy" [23] and CARIBU [24]. Further, there is a recent proposal to measure IYRs of ${ }^{252} \mathrm{Cf} \mathrm{SF}$ at CARIBU.

Nevertheless, the unique features in our implementation are broadband and high-resolving-power measurements in a non-scanning manner [21], fast extraction at the FRS-IC [15],fast mass measurement at the MR-TOFMS [21], and extraordinary cleanliness of the CSC [25]. The latter feature is the source for the essential element independence of the CSC, including comparison of a noble element (Rn), and a most reactive one (Th) [26].

These features will enable the derivation of FPY distributions of ground and isomer states, in addition to IYRs, which are anyway not affected by chemical dependence. The measured fragments half-lives may be as low as 10 ms, more than an order of magnitude shorter than in IGISOL [5] or CARIBU [24]). Furthermore, The CSC's high stopping ability and extraction efficiency, and compact gas-filled RFQ based beam line, will enable the measurement of FPYs and IYRs of rare fission products, even with a SF source in the range of tens of MBq.

The procedure for identifying, mass measuring and counting the amount of identified nuclides, including isomer-to-ground ratio calculations, and the correct estimation of the uncertainties, is described in detail in [27].

For SF studies, special internal CSC instrumentation is under design. It will include a short longitudinal DC field region $(\sim 50 \mathrm{~cm}$, rather than $100 \mathrm{~cm}$ for thermalizing projectile fragments), which will thermalize almost all SF fragments in the planned geometrical configuration. Its higher electric field will enable extraction times down to $\sim 10 \mathrm{~ms}$. It will further include a radioactive source holder for SF sources, which will include cone collimators at various opening angles, and foil degraders in front of the source that are optimized for maximal stopping of fission fragments in the buffer gas (Fig. 1).

For measuring FPYs at different times after the fission process, we will install a rotatable chopper in front of the source, with a wedge opening whose relative size will define the source's 'duty cycle'. The chopper's frequency sets the absolute durations in which the source is blocked. Synchronizing the chopper radial position with the voltages at the CSC extraction opening ('nozzle'), will enable recurrent extraction of fission products within the SF source 'pulse'. Lowering the chopper's frequency in synchronization with the CSC nozzle voltages, will enable recurrent extraction of fission products at longer times after the fission process, up to $\sim 10$ seconds [25].

The above temporal dependence, to be measured for the first time in this time range, will enable evaluation and benchmarking of procedures that are used today to de- 


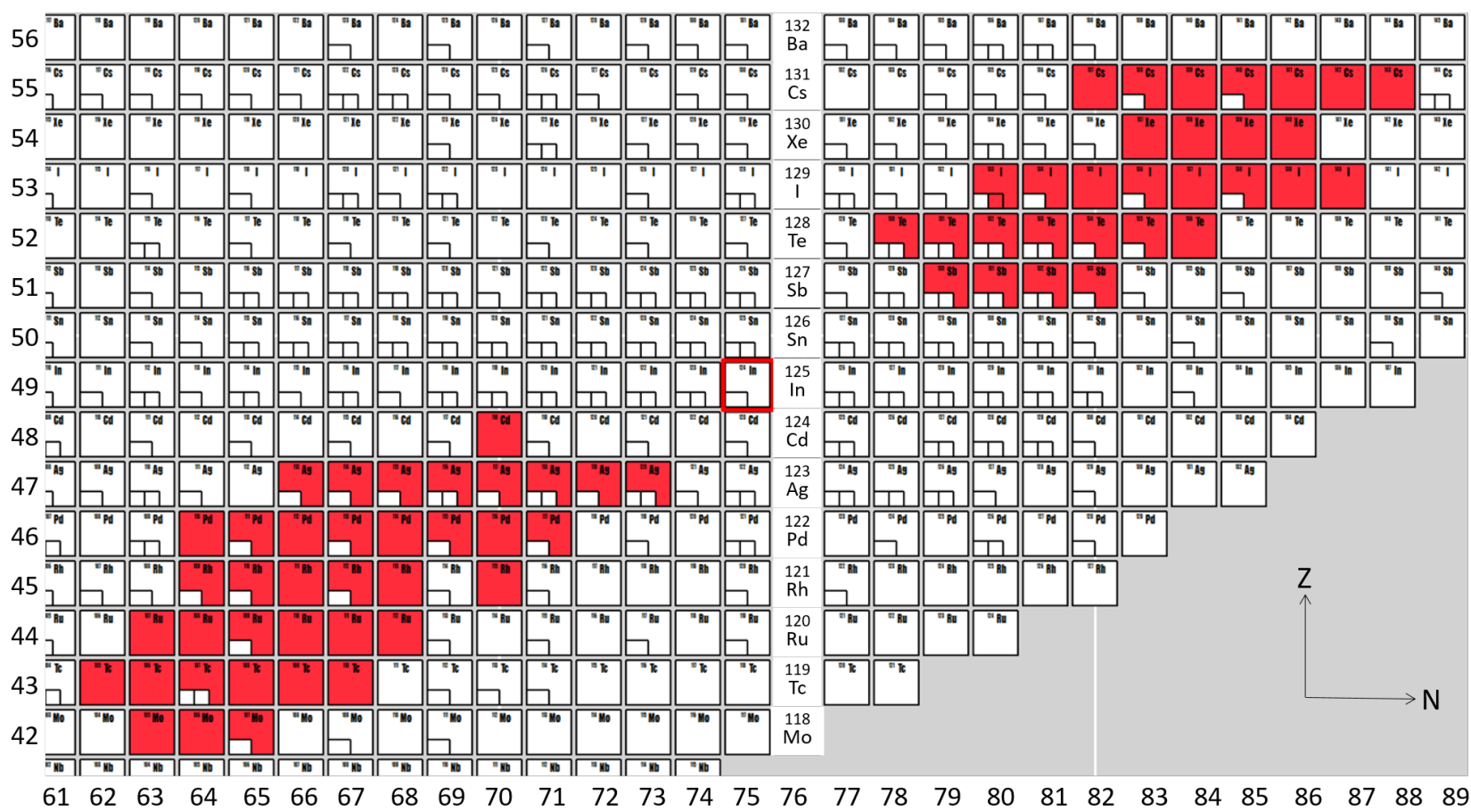

Figure 2. Section of the chart of nuclides. The red-marked nuclides are those measured in a method-demonstration run at the FRS$\mathrm{IC}$, with a $37 \mathrm{kBq}{ }^{252} \mathrm{Cf}$ SF source. Approximately 70 different fission fragments were identified and counted within a $\sim 50$ hour measurement period.

duce independent FPYs from measured cumulative FPYs [28]. Such procedures employ complex network calculations that depend strongly on the half-lives and $\beta$-delayed single- and multi-neutron emission branching ratios of short-lived neutron-rich fission products, whose uncertainties increase significantly for rarer fission products.

We intend to perform systematic measurements of various SF sources. The first ones will be a $10 \mathrm{MBq}{ }^{252} \mathrm{Cf}$ source and ${ }^{248} \mathrm{Cm}$. These measurements will extend understanding of FPY and IRY dependence on the nuclear structure of the fissioning isotope. Further, insights from this project will be invaluable input for future facilities and experiments that generate and study fission products, enabling optimal choices of proper fissioning nuclides and fission inducing reactions.

An example is the foreseen future facility for neutron induced fission at Phase II of the Soreq Applied Research Accelerator Facility (SARAF). It will use its especially high-flux neutron source in combination with an ion catcher system that includes a thin fissionable irradiation target within the CSC [29].

\section{First Demonstration of the Method}

The method was recently demonstrated with a low-activity $37 \mathrm{kBq}{ }^{252} \mathrm{Cf}$ SF source, installed in the existing FRS-IC with its standard DC cage. We operated the system for approximately 50 hours and identified and counted $\sim 70$ different fission fragments, with FPYs down to the level of $10^{-3}$, both in the low and high fission peaks. They are depicted in Fig. 2. Analysis of this data is ongoing, in or- der to extract coarse FPY values, as a preparation towards experiments with a high-activity $10 \mathrm{MBq}{ }^{252} \mathrm{Cf} \mathrm{SF}$ source.

\section{Summary}

We describe a novel method to measure FPYs and IYRs via direct simultaneous mass measurements in the FRSIC. We recently demonstrated the method with a lowactivity SF source at the existing CSC, identifying and counting fission fragments with FPYs as low as the level of $10^{-3}$. We are currently preparing a special DC cage for the CSC for measurements with a high-activity SF source, which will enable reaching fission yields several orders of magnitude lower, and studying the temporal dependence of FPYs and IYRs. This method could be used for systematic studies of various SF isotopes at the FRS-IC, and may set the stage for similar studies of neutron induced fission at the appropriate facilities.

\section{Acknowledgements}

This work was supported by the German Federal Ministry of Education and Research (BMBF) under under Contracts No. 05P16RGFN1 and No. 05P19RGFN1, by Justus-Liebig-Universität Gießen and GSI under the JLUGSI strategic Helmholtz partnership agreement, by HGSHIRe, and by the Hessian Ministry for Science and Art (HMWK) through the LOEWE Center HICforFAIR, by the European Union's Horizon 2020 research and innovation programme contract no. 654002 via the JRA SATNURSE, and by the Israel Ministry of Energy, Research Grant No. 217-11-023. 


\section{References}

[1] A.A. Sonzogni, E.A. McCutchan, T.D. Johnson, P. Dimitriou, Phys. Rev. Lett. 116, 132502 (2016)

[2] G. Martínez-Pinedo, D. Mocelj, N. Zinner, A. Kelić, K. Langanke, I. Panov, B. Pfeiffer, T. Rauscher, K.H. Schmidt, F.K. Thielemann, Progress in Particle and Nuclear Physics 59, 199 (2007), international Workshop on Nuclear Physics 28th Course

[3] P. Dimitriou, F.J. Hambsch, S. Pomp, IAEA INDC (NDS) 0713 (2016)

[4] P. Dimitriou, Private Communication (2018)

[5] I. Moore, T. Eronen, D. Gorelov, J. Hakala, A. Jokinen, A. Kankainen, V. Kolhinen, J. Koponen, H. Penttilä, I. Pohjalainen et al., Nuclear Instruments and Methods in Physics Research Section B: Beam Interactions with Materials and Atoms 317, 208 (2013), xVIth International Conference on ElectroMagnetic Isotope Separators and Techniques Related to their Applications, December 2-7, 2012 at Matsue, Japan

[6] H. Penttilä, D. Gorelov, V.V. Elomaa, T. Eronen, U. Hager, J. Hakala, A. Jokinen, A. Kankainen, P. Karvonen, I.D. Moore et al., The European Physical Journal A 52, 104 (2016)

[7] V. Rakopoulos, M. Lantz, A. Solders, A. Al-Adili, A. Mattera, L. Canete, T. Eronen, D. Gorelov, A. Jokinen, A. Kankainen et al., Phys. Rev. C 98, 024612 (2018)

[8] V. Rakopoulos, M. Lantz, S. Pomp, A. Solders, A. Al-Adili, L. Canete, T. Eronen, A. Jokinen, A. Kankainen, A. Mattera et al., Phys. Rev. C 99, 014617 (2019)

[9] P. Armbruster, M. Asghar, J. Bocquet, R. Decker, H. Ewald, J. Greif, E. Moll, B. Pfeiffer, H. Schrader, F. Schussler et al., Nuclear Instruments and Methods 139, 213 (1976)

[10] A. Bail, O. Serot, L. Mathieu, O. Litaize, T. Materna, U. Köster, H. Faust, A. Letourneau, S. Panebianco, Phys. Rev. C 84, 034605 (2011)

[11] T. Wang, G. Li, L. Zhu, O. Hen, G. Zhang, Q. Meng, L. Wang, H. Han, H. Xia, Phys. Rev. C 96, 034611 (2017)

[12] H.H. Knitter, F.J. Hambsch, C. Budtz-Jørgensen, Nuclear Physics A 536, 221 (1992)

[13] G. Mariolopoulos, C. Hamelin, J. Blachot, J. Bocquet, R. Brissot, J. Crançon, H. Nifenecker, C. Ristori, Nuclear Physics A 361, 213 (1981)

[14] K.H. Schmidt, B. Jurado, C. Amouroux, C. Schmitt, Nuclear Data Sheets 131, 107 (2016), special Issue on Nuclear Reaction Data

[15] W.R. Plaß, T. Dickel, S. Purushothaman, P. Dendooven, H. Geissel, J. Ebert, E. Haettner, C. Jesch, M. Ranjan, M.P. Reiter et al., Nucl. Instrum. Methods B 317, 457 (2013)

[16] H. Geissel, P. Armbruster, K. Behr, A. Brünle, K. Burkard, M. Chen, H. Folger, B. Franczak,
H. Keller, O. Klepper et al., Nucl. Instrum. Methods B 70, 286 (1992)

[17] M. Ranjan, S. Purushothaman, T. Dickel, H. Geissel, W.R. Plaß, D. Schäfer, C. Scheidenberger, J.V. de Walle, H. Weick, P. Dendooven, Europhys. Lett. 96, 52001 (2011)

[18] S. Purushothaman, M.P. Reiter, E. Haettner, P. Dendooven, T. Dickel, H. Geissel, J. Ebert, C. Jesch, W.R. Plaß, M. Ranjan et al., Europhys. Lett. 104, 42001 (2013)

[19] M. Ranjan, P. Dendooven, S. Purushothaman, T. Dickel, M. Reiter, S. Ayet San Andrés, E. Haettner, I. Moore, N. Kalantar-Nayestanaki, H. Geissel et al., Nucl. Instrum. Methods A 770, 87 (2015)

[20] E. Haettner, W.R. Plaß, U. Czok, T. Dickel, H. Geissel, W. Kinsel, M. Petrick, T. Schäfer, C. Scheidenberger, Nucl. Inst. Methods A 880 (2018)

[21] T. Dickel, W.R. Plaß, A. Becker, U. Czok, H. Geissel, E. Haettner, C. Jesch, W. Kinsel, M. Petrick, C. Scheidenberger et al., Nucl. Instrum. Methods A 777, 172 (2015)

[22] T. Dickel, W.R. Plaß, S. Ayet San Andrés, J. Ebert, H. Geissel, E. Haettner, C. Hornung, I. Miskun, S. Pietri, S. Purushothaman et al., Phys. Lett. B 744, 137 (2015)

[23] C. Düllmann, B. Eichler, R. Eichler, H. Gäggeler, D. Jost, U. Kindler, D. Piguet, S. Soverna, P. Thörle, N. Trautmann et al., Nuclear Instruments and Methods in Physics Research Section A: Accelerators, Spectrometers, Detectors and Associated Equipment 512, 595 (2003)

[24] R.C. Pardo, G. Savard, R.V.F. Janssens, Nuclear Physics News 26, 5 (2016)

[25] I. Miskun, T. Dickel, I. Mardor, C. Hornung, D. Amanbayev, S. Ayet San Andrés, J. Bergmann, J. Ebert, H. Geissel, M. Górska et al., Accepted for publication in Eur. Phys. Jour. A (2019), https://arxiv.org/abs/1902.11195

[26] W.R. Plaß, T. Dickel, I. Mardor, S. Pietri, H. Geissel, C. Scheidenberger, D. Amanbayev, S. Ayet San Andrés, J. Aysto, D. Balabanski et al., Hyperfine Interactions (2019), https://doi .org/10.1007/s10751-019-1597-4

[27] S. Ayet San Andrés, C. Hornung, J. Ebert, W.R. Plaß, T. Dickel, H. Geissel, C. Scheidenberger, J. Bergmann, F. Greiner, E. Haettner et al., Phys. Rev. C 99, 064313 (2019)

[28] M. Lammer, H. Denschlag, T. England, A. Goverdovski, M. James, Q. Liang, T. Liu, R. Mills, B. Rider, G. Rudstam et al., IAEA-TECDOC-1168 (2000)

[29] I. Mardor, O. Aviv, M. Avrigeanu, D. Berkovits, A. Dahan, T. Dickel, I. Eliyahu, M. Gai, I. GavishSegev, S. Halfon et al., The European Physical Journal A 54, 91 (2018) 